Cahiers de recherches médiévales

\title{
De quelques figures féminines dans les Faits des
} Romains

\section{Catherine Croizy-Naquet}

\section{(2) OpenEdition \\ 1 Journals}

Édition électronique

URL : https://journals.openedition.org/crm/2509

DOI : 10.4000/crm.2509

ISSN : 1955-2424

Éditeur

Honoré Champion

Édition imprimée

Date de publication : 12 décembre 1996

Pagination : 201-220

ISSN : 1272-9752

\section{Référence électronique}

Catherine Croizy-Naquet, " De quelques figures féminines dans les Faits des Romains », Cahiers de recherches médiévales [En ligne], 2 | 1996, mis en ligne le 04 février 2008, consulté le 15 décembre 2022. URL : http://journals.openedition.org/crm/2509; DOI : https://doi.org/10.4000/crm.2509 


\section{ARM}

\section{De quelques figures féminines dans les Faits des Romains}

Parmi les récits historiques en prose qui voient le jour au $\mathrm{XII}^{\mathrm{e}}$ siècle se distingue une histoire ancienne anonyme, les Faits des Romains, consacrée pour l'essentiel à la vie de César' . Comme l'indique le titre complet de l'ouvrage ${ }^{2}$, le texte est une compilation effectuée d'après plusieurs cuvres antiques: la Vie de César de Suétone, la Conjuration de Catilina de Salluste, la Pharsale de Lucain et les Commentaires de la guerre des Gaules de César auxquels s'ajoutent d'autres sources dont l'identification n'est pas toujours aisée ${ }^{3}$. Son succès fut considérable tout au long du Moyen Age, comme en témoigne le grand nombre de manuscrits, une soixantaine environ ${ }^{4}$. Il est vrai que la transcription en prose française offre une fresque ample et très vivante de l'histoire ancienne. La thématique de l'œuvre est essentiellement politique et guerrière puisqu'y est relatée la conquête du pouvoir par César. Dans un tel contexte, les figures masculines sont dominantes, les plus marquantes étant celle de Vercingétorix, puis celles de Caton et de Pompée, envisagées généralement en fonction du héros principal qu'est César ${ }^{5}$. En dépit du peu de place accordée à la femme, trois figures féminines émergent cependant dans cet univers d'hommes : Marcia, l'épouse de Caton, Cornélia, l'épouse de Pompée, et Cléopâtre, la sceur incestueuse de Ptolémée, le roi d'Égypte. Les examiner présente l'intérêt de voir comment le compilateur travaille ses sources, sa fidélité à leur égard et, en même temps, la distance qu'introduisent formellement l'usage de la

\footnotetext{
${ }^{1}$ Les Faits des Romains, éd. L. -F. Flutre et K. Sneyders de Vogel, 2 vol., Paris-Groninge, 1938. Le projet initial de l'auteur était de raconter en douze livres l'histoire romaine, de la naissance miraculeuse de César à la fin du règne de Domitien, mais il n'a composé que la vie de César pour des raisons que nous ignorons. Pour une présentation de l'œuvre, voir P. Meyer, "Les premières compilations françaises d'histoire ancienne, I. Les Faits des Romains, II. Histoire ancienne jusqu'à César », Romania, t. 14, 1885, pp. 1-85.

${ }^{2}$ Le titre complet de l'ouvrage est Li Fet des Romains compilé ensemble de Saluste et de Suetoine et de Lucan.

${ }^{3}$ Sur la variété des sources utilisées, sources directes et indirectes, et leur identification, voir éd. L. $-F$. Flutre et K. Sneyders de Vogel, t. 2, pp. 22-29. Dans cet article, nous utiliserons, pour La Guerre civile (la Pharsale), l'édition et la traduction d'A. Bourgery et de M. Ponchont, Paris, Société "les Belles Lettres ", t. 1-2, 1948.

${ }^{4}$ Cf. L. -F. Flutre, Les manuscrits des Faits des Romains, Paris, Hachette, 1932, et Li Fait des Romains dans les littératures française et italienne du XIIf siècle au XVf siècle, Paris, Hachette, 1932. Sur le public concerné par les histoires sur l'antiquité et sur l'usage plus tardif des Faits des Romains, voir aussi J. Monfrin, "Les traducteurs et leur public en France au Moyen Age », L'humanisme médiéval dans les littératures romanes du XIf au XIIf siècle, éd. A. Fourrier, Paris, 1964, pp. 247-264. Et sur le cas spécifique des Faits des Romains, cf. B. Guenée, «La culture historique des nobles: le succès des Faits des Romains (XIV'-XV siècles)", La noblesse au Moyen Age Essais à la mémoire de Robert Boutruche, réunis par Ph. Contamine, Paris, 1976, pp. 261-288. D'après l'étude des manuscrits, de leur localisation et de leur diffusion, l'historien montre notamment que l'œuvre a d'abord été écrite pour le milieu universitaire parisien, avant d'être reque par le public läque cultivé, art. cit., pp. 263-267.

${ }^{5}$ Le récit est composé de plusieurs parties. La première concerne la période située avant la guerre des Gaules, la deuxième est consacrée à la guerre des Gaules et fait intervenir la figure de Vercingétorix, la troisième, où Caton et Pompée occupent une place majeure, porte sur la guerre civile, et la dernière relate l'apogée de César et sa mort, avec un ultime passage sur la personnalité du héros romain.
} 
prose française et « idéologiquement» une manière de considérer la femme. Se profile ainsi une méthode de travail associée à des desseins historiques marqués de l'époque.

\section{La représentation de trois figures féminines}

L'importance quantitative et les modes d'intervention des figures féminines dans le texte varient selon l'héroïne et selon son rôle dans l'économie du récit. Il n'est ainsi question qu'une seule fois de Marcia, ex-épouse et à nouveau épouse de Caton, quand le compilateur établit les circonstances de la guerre civile et explique la position de Caton en faveur de Pompée. Entre une conversation avec Brutus à ce sujet et un portrait de Caton, l'auteur intercale, à la suite de Lucain, un passage consacré à Marcia, dont il fait une rapide présentation avant de lui laisser la parole ${ }^{6}$. Apparemment sans nécessité particulière pour le déroulement du récit - Marcia demande à Caton de finir ses jours à ses côtés - , cet intermède semble avoir troublé le compilateur, comme le montre sa maladresse à l'insérer dans la narration. Après le portrait de Marcia, entrepris sans transition au terme du dialogue entre Caton et Brutus et qui introduit un retour au passé, l'auteur revient au temps du récit de manière plus artificielle que dans la source:

En ce que Catons et Brutes parloient en tel maniere, Marcia vint a l'uis Caton et hurta por entrer. (p. 369, 1. 19-20)

Chez Lucain, ce bref passage précédait l'histoire de Marcia, ce qui rendait l'intervention de l'héroïne plus naturelle?

L'unique intervention de Marcia souligne davantage la présence récurrente de Comélia, dans la partie qui porte sur à la guerre civile. L'épouse de Pompée est mentionnée une première fois lorsque le traducteur relate le départ de Pompée à l'issue de la bataille de Brindisi (p. 386, 1. 14), puis lorsqu'il évoque le songe du chef romain qui voit sa première femme défunte lui reprocher son mariage hâtif avec Comélia :

«Tu [Pompée] m'eüs molt tost obliee por Cornille; ele n'atendi gaires a toi espouser $»($ p. 387, 1. 8-9)

Ces deux rapides mentions, outre qu'elles indiquent la place importante de Comélia dans l'existence de Pompée, anticipent les événements à venir, d'une part les situations de fuite et d'exil des deux héros et, d'autre part, le sort fatal de Pompée dont Comélia s'attribue la responsabilité, confirmant les prédictions de Julia ${ }^{8}$. Plus avant dans le texte, l'intervention de Comélia est quasiment continue, sa destinée étant intimement liée à celle de Pompée par le mariage. Le compilateur dépeint le couple dans la réalité de sa vie conjugale :

Un suer gisoient en lor lit et dormi orent tote la nuit. Endroit l'ajornement il furent esveillié. Comille ot embraciee Pompee et le vost baisier. (p. 471, 1. 27-30)

\footnotetext{
${ }^{6}$ Voir éd cit., pp. 369-371 ; Lucain, t. 1, II, 326-376.

${ }^{7}$ Lucain, II, 326-329.

${ }^{8}$ Pour les prédictions/accusations de Julia, cf. op. cit. pp. 386-387.
} 
Mais surtout, il donne souvent la parole à Cornélia, dans des dialogues avec Pompée ou dans des discours qui tournent au monologue, par exemple quand elle assiste de loin au meurtre de son mari :

« Beau sire, lessiez me vos por ce arriere en ceste nef que vos moreüssez sanz moi ? » (p. 565, 1. 19-20)

Le portrait de Cornélia se construit à travers les différentes scènes dans lesquelles elle joue un rôle plus ou moins passif, et grâce au discours direct dont elle bénéficie largement.

Avec Cléopâtre, la troisième figure féminine marquante des Faits des Romains à apparaître dans le texte, l'auteur modifie quelque peu ses modalités de présentation. Cléopâtre est en effet omniprésente dans l'épisode relatant la présence de César en Égypte ${ }^{9}$, aussi bien par le portrait moral et physique dont elle est l'objet que par ses prises de parole et ses actes. Le portrait moral n'est en réalité qu'ébauché par les remarques de Lucain, reprises ou développées par le traducteur:

Ele se fu apareilliee au plus bel que ele onques pot por plere a Cesar et por enlacier le et atrere a sa volenté et a s'amor. (p. 623,1.15-17)

ou bien par les jugements des personnages masculins, tel celui, explicite, de Photin:

« la maligne suer [Cleopâtre] est ja jointe a son frere a noces, por estre dame et por mesmener le regne » (p. 634, 1. 22-23)

ou celui, implicite, de César dans l'hommage rendu à sa beautét ${ }^{10}$. En revanche, le portrait physique est l'occasion d'une longue pause descriptive au cours de laquelle le compilateur dépeint exhaustivement la beauté de la reine et la recherche de sa parure. En parallèle, et peut-être en guise d'illustration ou d'explication ${ }^{11}$, il relate ses diverses actions, de sa fuite de la prison où Ptolémée, à l'instigation de Photin, l'avait enfermée, à ses intrigues pour rendre le pouvoir à son frère et se l'octroyer enfin à elle-même ${ }^{12}$.

A l'évidence, les trois figures féminines ne sont pas traitées pareillement. Le rôle de la première dans la narration est nul; celui de la deuxième dépend de celui de Pompóe à qui son sort est lié; quant à celui de la troisième, il est beaucoup plus déterminant puisque les actions de Cléopâtre décident en partie des décisions politiques et militaires de César. De ces interventions variées découlent trois types de femme ${ }^{13}$ et trois manières d'être femme, dans le mariage et hors mariage, distinction qui oppose nettement les deux premières figures à la troisième ; encore faudra-t-il sans doute nuancer pour Cornélia.

\footnotetext{
${ }^{9}$ Voir op. cit, pp. 621-657; Lucain, X, 1-546. Le compilateur a dû recourir aussi à des sources diverses, le récit de Lucain étant inachevé et incomplet. Il utilise notamment les Vies des douze Césars, de Suétone, pour lesquelles nous utilisons l'édition et la traduction de H. Ailloud, Paris, Société « les Belles Lettres », 1967, t. 1, livre I Sur les différentes utilisations faites par le compilateur, cf. éd. cit., t. 2, pp. 207-210.

${ }^{10}$ Voir éd. cit., p. 623, 1.22 et ss. et surtout p. 625, 1. 12 et ss.

${ }^{11}$ Le portrait explique entre autres et la conduite de Cléopâtre qui use de sa beauté pour parvenir au pouvoir, et la conduite de César qui cède devant son charme. Sur le rôle des portraits, cf. Ed. Faral, Les arts poétiques du XIf et du XIff siècle, Paris, lère éd., 1923, p. 74 et ss.

${ }^{12}$ Ed. cit, pp. 623-628.

${ }^{13} \mathrm{Ou}$ bien trois types de femmes impliquent trois types d'intervention.
} 
Marcia est d'emblée présentée comme un modèle d'épouse dont les qualités majeures sont la soumission à son époux, sa vertu et son souci de l'honneur. L'histoire de sa vie en est une illustration éloquente. Mariée d'abord à Caton, celui-ci la quitte:

Quant il fu de tel aage que il ne pooit mes engendrer et ele pooit encore anfanz porter, (p. 369, 1. 6-7) ${ }^{14}$.

Pour obéir et malgré ses réticences, elle épouse alors Hortensius de qui elle a d'autres enfants, avant de revenir auprès de son premier époux, à la mort du second, pour sauvegarder son honneur:

« Ge voill [...] que cil qui apres nestront ne soient pas en dote savoir mon se tu me gitas de toi por ma puterie quant Hortensius m'espousa, ou se tu me donnas a lui de ton bon gré, si con l'en doit prode fame doner por anfanz avoir. " (p. 370, 1. 6-10)

Dans ce parcours, la vocation de la femme est clairement définie : elle est uniquement destinée à procréer, l'union conjugale n'ayant pour fonction que d'assurer la descendance et la pérennité de la lignée. Le retour auprès de Caton traduit l'autre aspect du mariage, celui du partage: la femme sert et soutient son mari en toute situation:

" ainz voill estre parçonniere de ta paine et de ton travaill." (p. 370, 1. 12-14)

Selon Caton et donc selon Marcia, la femme n'existe qu'en tant que mère et compagne. La dimension sexuelle est exclusivement guidée par les impératifs du lignage à assurer. Toute forme de séduction ou toute tentative de séduction est déconsidérée selon les principes de Caton. Lorsque Marcia se présente devant Caton:

Ne se fu pas paree ne pingniee [...]. Ele pensoit bien : autrement ne pleüst ele pas a Caton, qui tant estoit prodom, se ele venist paree de la fosse son mari. (p. 369, 1. 2328)

A sa manière, Cornélia est aussi un modèle d'épouse, avec cette différence fondamentale qu'à un amour de raison, elle oppose un amour absolu dans lequel la dimension amoureuse et sensuelle a une place centrale. Dans la relation conjugale qui l'unit à son mari, l'aspect de séduction semble négligé - ou dépassé - , du moins la séduction par l'apparence ${ }^{15}$. Aussi l'héroïne n'est-elle perçue physiquement que dans ses manifestations de désespoir:

Quant les puceles connurent vraiment que ce estoit lor sires, eles conmencierent a crier et voloient lor dame relever de pasmoison; mes eles ne pooient, car ele gisoit conme morte. (p. 547, 1. 10-13)

Le compilateur insiste sur les sentiments réciproques qu'éprouvent les deux époux l'un envers l'autre:

car mout s'entramoient de grant amor. (p. 471, 1.25)

\footnotetext{
${ }^{14}$ La raison invoquée pour la séparation n'est pas entièrement convaincante dans la perspective d'un ascétisme stö̈que. Caton quitte Marcia au moment où il perd tout appétit sexuel.

${ }^{15}$ Point ou peu de remarques sur sa beauté. S'il y a entreprise de séduction, celle-ci s'effectuerait par les larmes et les lamentations pour susciter la pitié et obtenir ainsi de ne pas être séparée de son mari. Cf. éd. cit., p. 474.
} 
Cornélia est le type de femme qui combine amour et mariage, qui définit sa conception du mariage en fonction de l'amour qu'elle porte à son époux. De fait, elle n'envisage pas le mariage dans l'absence de partage ni dans la séparation:

« Ne place Dieu que tu soies en aversité, et je ne soie parçonniere de ta mesestance [ou que je soie]. [...] Se tu as bien, je avrai bien autresi ou que je soie ; se tu as mal, je avrai mal. " (p. 473, 1. 16-21)

Dans les dialogues, ce portrait de femme mariée et amoureuse se précise et se nuance. Son intransigeance cède devant les ordres de son mari : elle finit par accepter le départ pour l'île de Lesbos:

Ot ele einsi parlé, elle sailli tote forsenee jus del lit et dit par grant corroz qu'ele n'atendroit plus, ainz s'en iroit. (p. 474, 1. 25-26)

Une clause implicite de soumission entre donc aussi dans le contrat de leur union. A la conception d'un partage total s'ajoute celle de la soumission à l'époux. Au fil du récit, Comélia éprouve l'inadéquation de ces deux principes qui peuvent se révéler dans les faits contradictoires. Au regard de Marcia, c'est un personnage beaucoup plus complexe avec ses craintes et avec des certitudes qui se heurtent aux réalités politiques et militaires. Dépeinte dans le déroulement de sa vie conjugale liée au sort de Pompée, elle conserve toujours les mêmes sentiments amoureux et la même exigence de partage, lors même que sa souffrance, déclinée sous tous ses aspects, est appréhendée dans ses incidences sur sa conduite. Contrairement à Marcia, elle est un personnage pathétique par sa condition de femme amoureuse et de femme mariée, comme si les exigences de l'amour et du mariage se révélaient incompatibles ou impuissantes devant un sort contraire. Cornélia est une figure de l'amour conjugal voué à l'échec et à la mort.

En parfait contraste avec Marcia et Cornélia, Cléopâtre incarne un autre type de femme, celui de la femme fatale. Dans le portrait moral qui s'élabore au fur et à mesure que se déroule l'épisode, Cléopâtre est présentée continûment comme une femme perverse, ensorceleuse et corruptrice d'homme, et ce, dès qu'elle joue un rôle véritable dans le récit, c'est-àdire en prison:

Lors fist tant qu'ele corrompi et deçut celui qui la devoit garder par beles paroles et li promist granz richeces et granz honors se ele james pooit venir a terre. (p. 623, 1. 711)

C'est surtout auprès de César que s'exerce son pouvoir de séduction. Elle en connait tous les artifices, par exemple l'affectation de tristesse pour attendrir l'homme:

Ele se fist mate chiere et triste au plus qu'ele pot. Tote fu eschevelee et atomee a guise de fame qui devoit merci crier et requerre. (p. 624, 1. 11-13) 
Comuption qui débouche pour César sur l'adultère ${ }^{16}$. Cléopâtre est également discréditée par l'un de ses ennemis, Photin, qui souligne ses mœurs honteuses, l'inceste et la bigamie ${ }^{17}$. Sa conduite est en fait guidée par sa soif de pouvoir et de domination:

«Ele va ja corant entre II. mariz; li uns [Ptolémée] li a donee Egypte, li autre Rome." (p. 634, 1. 24-25)

Ainsi décrite, la reine est un personnage emblématique du mal absolu. Et son portrait physique, au lieu d'atténuer la charge négative de l'éthopée, la conforte. Si l'héroïne a tous les traits d'une beauté absolue, il s'agit d'une beauté étudiée et calculée. A son charme naturel, elle ajoute tous les atouts du maquillage et du fard pour réussir à séduire César :

Cleopatra refu ilec, qui se pena de sa mercerie desploier por escomovoir Cesar a luxure, por mielz fere sa besoigne. (p. 625, 1. 17-18)

Le traducteur use d'expressions familières dont l'équivalent, on le verra, n'est certes pas chez Lucain, manière de rendre plus éloquente la propension à ensorceler de la reine ${ }^{18}$. La dimension sexuelle et sensuelle est constitutive du personnage de Cléopâtre. A l'instar de Lucain, le compilateur évoque d'ailleurs l'acte charnel avec un jugement réprobateur, à tout le moins sans le lier à un sentiment amoureux véritable ${ }^{19}$. Au final, le portrait est apparemment sans nuance: Cléopâtre est l'incamation féminine du mal et l'image que Lucain et son traducteur donnent d'elle est celle d'une prostituée:

Tant s'estoit Cleopatra beau paree, que bel avival de puterie et de luxure avoit en li. (p. 627, 1. 9-10)

aux antipodes d'une Marcia dont l'image est, à l'inverse, celle de la mère. Entre les deux, Cornélia peine à trouver sa place. Épouse et mère, elle est à l'image de Marcia; femme amoureuse et aimante, elle a quelque ressemblance avec Cléopâtre dans le trouble et la remise en question qu'elle suscite chez Pompée, partagé entre amour et devoir.

Les composantes des différents portraits féminins sont confortées et parachevées par les représentations masculines. A un type de femme correspond un type d'homme et la description de l'une peut servir de faire-valoir à l'autre, ou mieux l'homme peut trouver en la femme son double. La symbiose la plus parfaite s'opère dans le couple formé par Caton et Marcia. Les qualités de l'époux sont celles de l'épouse, ce qu'illustre le portrait de Caton qui suit immédiatement celui de Marcia. Comme Caton est preudom ${ }^{20}$, Marcia est vertueuse et soucieuse de l'honneur: c'est l'une des raisons de son attachement pour son mari :

" onques ne fis rien contre ton conmandement ne contre t'onor, ne ne fu mauvese novele oïe de moi. » ( p. 369, 1.31-32)

\footnotetext{
${ }^{16}$ Ce qui suscite la vive condamnation du compilateur à la suite de Lucain, condamnation qui révèle les valeurs morales qu'il cherche à transmettre, comme la fidélité conjugale incarnée par le couple Caton/Marcia et le couple Pompée/Comélia.

${ }^{17}$ Voir éd. cit., pp. 634-635, [26].

${ }^{18}$ Cf. Lucain, X, 104135.

${ }^{19}$ Voir éd. cit., p. 623,1.21-23; p. 634, 1. 22 et ss. ; p. 638, 1. 45.

${ }^{20}$ Ed. cit. p. 369, 1. 14, 1. 28. Ce terme serait l'une des manières de désigner le comportement de Caton, fondé sur la recherche et/ou la pratique de la virtus propre aux stoïciens, avec une évidente christianisation.
} 
Comme Caton, elle aime et pratique la chasteté :

Et merveille estoit il ne touchast ja a fame charnaument nule foiz, se ne fust por enfant engendrer, ainz contrestoit vertueusement as aguillons de luxure. Marcia sa fame ne ramoit pas meins chasteé. (p. 371, 1. 24-28)

Double féminin, Marcia est aussi le faire-valoir de Caton. Elle lui donne l'occasion de montrer sa grandeur d'âme en la recevant à nouveau comme épouse (p. 370, l. 18-21). La parfaite entente entre les deux personnages et leur mode de relation sont rédupliqués ou emblématisés par la description de l'attitude de Caton envers Rome:

Il estoit peres et mariz a la cité, car il li porveoit et aidoit come li pere fet sen anfant o li mariz a sa fame. (p. 371, 1. 21-22)

Conduite politique et conduite privée se fondent l'une dans l'autre, ou du moins convergent, ne laissant nulle interrogation sur le devoir à suivre, nulle nécessité de choisir entre l'attachement à une femme et l'attachement à une ville et/ou à une cause. Le portrait exclusivement moral de Marcia ${ }^{21}$, sans incidence sur le récit, n'est qu'une manière d'approfondir le portrait de son époux et de montrer aussi les conditions qui fondent l'équilibre d'un couple, présenté comme l'accomplissement de l'idéal du mariage au Moyen Age ${ }^{22}$.

Avec Cornélia et Pompée, les données se modifient sensiblement. Au regard du couple précédent, la figure féminine est nettement plus affirmée et individualisée, elle existe par elle-même, par ses sentiments, et pas seulement comme adjuvant de Pompée. Entre les deux époux s'observent bien des similitudes susceptibles de créer une communion d'intentions et de pensées. Tous deux éprouvent au même degré, semble-t-il, le sentiment amoureux (p. 471, 1. 25). Cette intensité de l'amour se traduit notamment par l'attention portée à l'autre. Pompée se soucie du sort de Comélia :

Il voloit que la dame fust la a seür; que que il avenist de la bataille. (p. 471, 1. 21-22)

comme Comélia se soucie de celui de Pompée :

La dame, qui l'atendoit, n'estoit gaires a aise. Ele estoit en grant cure et en grant soign, ne dormoit gaires totes les nuiz; sis cuers estoit en Thessaille. (p. 546, 1. 2628)

Les souffrances que leur cause la séparation sont identiques:

Il n'avoient onques eü si tristre jor en lor vie come il orent ce jor que il departirent. (p. 474, 1. 30-31)

Les deux époux sont par ailleurs conscients du rang qu'ils occupent et des nécessités de conduite que cela implique ${ }^{23}$. L'union conjugale est faite d'obligations que l'un et l'autre respectent. Comélia sait par exemple qu'elle a mission à la mort de Pompée de transmettre à

\footnotetext{
${ }^{21}$ Déjà chez Matthieu de Vendôme, Marcia est l'incamation des qualités morales et n'est l'objet d'aucume notation sur son apparence; cf. Ars versificatoria in Ed. Faral, op. cit., p. 128.

${ }^{22}$ Cf. J. M. A. Beer, A Medieval Caesar, Études de Philologie et d'Histoire, 30, Geneva, 1976, p. 178.

${ }^{23}$ Voir en particulier p. 547, [4].
} 
son fils le testament de son mari ${ }^{24}$. Entre les deux êtres règnent une profonde harmonie de sentiments et une compatibilité affective et sociale.

Mais Cornélia n'est pas exactement le double féminin de Pompée. Elle est plus vraisemblablement un faire-valoir, en ce qu'elle montre le profond amour que peut susciter un homme de sa valeur. Elle ne correspond pas en effet totalement au portrait de la femme idéale que brosse Pompée lorsqu'il la rejoint à Lesbos. Il lui prône la modestie et l'acceptation d'un sort contraire en la personne d'un mari vaincu ${ }^{25}$. Curieusement, il reproche à Cornélia une peine qu'elle éprouve uniquement pour sa mauvaise condition physique, et non pour le revers de fortune qui le frappe. Dans ce même discours, il définit les devoirs de la fermme dans l'adversité:

« Se vos lessiez tot por moi suivre, por estre povre avec moi, ja mes ceste renomee ne vos faudra. Fame ne doit pas doloser ne plorer tant con ses barons est vis, ses lermes ne sont pas covenables ; quant il est morz, lors proimes doit ele plorer. " (p. 548, 1. 711)

privilégiant le caractère social du mariage au détriment de l'aspect sentimental et intime. Une différence d'appréciation et peutêtre de logique apparaît entre les deux personnages: Cornélia évolue dans la sphère privée, en dépit d'un sens de l'honneur aigu, et Pompée dans la sphère publique, à moins qu'il ne soit partagé entre les deux et s'efforce de les concilier. La belle harmonie qui caractérise le couple est minée insidieusement par une divergence fondamentale quant à la place de l'amour. Du reste, la fusion des deux personnages est rompue par la séparation de l'exil, puis par la mort de Pompée dont Comélia se rend responsable. Cet aveu de l'héroïne est sans doute l'une des manières de désigner l'impossibilité de l'amour absolu à être dans le mariage.

La complémentarité qui s'établit entre Cléopâtre et César est d'une autre nature. Il était logique que ces deux héros se rencontrassent. Cléopâtre est à maints égards le double féminin de César. Comme lui, elle est avide de pouvoir et prête à tout pour y parvenir. Si César recourt à la force, voire à la cruauté, elle recourt, elle, aux moyens dont elle dispose en tant que femme, sa beauté, sa séduction et son intelligence. Elle séduit César, non par ses propos, mais par sa beauté :

Cleopatra n'eüst de rien amolloiees les dures oreilles Cesar par sa proiere, mes sa face et sa biautez firent sa besoigne. (p. $625,1.10-12$.)

Comme César, elle est dépourvue de sens moral : elle est la femme de plusieurs hommes de même que César est l'homme de plusieurs femmes ${ }^{26}$. Tous deux ne craignent ni luxure ni débauche $^{27}$. La scène du festin dans laquelle elle figure avec César est, à ce titre, tout un

${ }^{24}$ Cf. éd. cit, les propos de Comélia à Sextus, p. 580, 1. 14-26.
${ }^{25}$ Ed. cit, p. $548,1.1-17$.
${ }^{26}$ Les amants/maris de Cléopâtre sont mentionnés, au même titre que les maîtresses de César, maîtresses
contraintes (César a violé Postumia, Lollia, Tertullia, Mucia etc. op. cit., p. 721 et ss) ou consentantes
(dont Eunoé, l'épouse du roi Bogudis, op. cit, p. 659 et ss.).
${ }^{27}$ Dans le portrait de César qui clôt le texte, l'auteur s'attarde d'ailleurs sur les moeurs et la luxure de
César: « De quel luxure Cesar fu et de ses mors ", p. 721 . Voir, à ce sujet, dans Romancing the Past:
the Rise of Vemacular Prose Historiography in Thirteenth Century France, Berkeley et Los Angeles,
1993, l'analyse de G. Spiegel, qui voit dans l'union des deux héros « a regressive surrender to 
symbole. Organisatrice des festivités, déployant un luxe ostentatoire à l'égal de celui de sa parure, elle émeut César et le touche dans ses sens, dans ses goûts et dans ses appétits :

Et embrasoient en ceste guise Ptolemez et Cleopatra les cuers de lor hostes, qui n'aloient le monde conquerant ne destruant fors por avoir seignorie et richece. (p. $627,1.16-18)$

Image du mal au féminin, Cléopâtre est le double de César, image du mal au masculinn ${ }^{28}$. Les deux héros sont les faire-valoir l'un de l'autre, et se trouvent sur un pied d'égalité, contrairement à ce qui se passe dans les couples précédents, où la femme est soumise aux décisions de son époux. La reine mène une action politique comparable à l'action militaire que mène César, mais avec des moyens différents. Cette capacité fait d'elle un allié pour César qui traite avec elle comme avec un homme. Unique, et non des moindres, concession à l'amour ou plutôt à l'attirance sexuelle ${ }^{29}$, il lui laisse l'Égypte à gouvemer au lieu d'en faire une province:

Et ne fust por amor de li [Cléopâtre], il eüst fet province dou regne d'Egypte, - ce est terre sanz roi-, et i eüst mis un prevost procureor, qui fust Romains, a cui li Egyptien respondissent por Rome. (p. 656, 1. 20-23)

Les trois couples se caractérisent par une concordance entre les figures féminines et masculines dépeintes. Les portraits de Marcia et de Comélia ne peuvent d'ailleurs se concevoir ni se construire qu'en fonction d'un homme et/ou d'un sentiment amoureux. Pourtant, la complémentarité n'est pas toujours totale, le couple le plus solide étant celui où la dimension amoureuse et sexuelle est inexistante. En contrepoint, celui de Pompée et de Cornélia est fragilisé par ce qui est aussi sa force, l'intensité du sentiment amoureux. Illustration de l'amour ou de l'attirance hors mariage, le dernier couple est peut-être le plus abouti, celui où la femme, active et sûre d'elle-même, s'impose comme l'autre de l'homme, non pas son ombre mais son égal. C'est elle cependant qui est constamment blâmée et dépréciée. Le compilateur donne ainsi à voir trois figures féminines, au rôle variable et aux personnalités plus ou moins bien individualisées, le tout assorti d'un jugement moral. Dans ces portraits, il dit suivre sa source principale, Lucain, se comporter en simple traducteur et médiateur entre le texte latin et la prose vernaculaire.

\section{Le travail et les desseins du compilateur}

Indubitablement, l'auteur affiche une remarquable fidélité à sa source latine. Dans l'ensemble de l'ouvrage, il montre par le recours au style indirect ou par l'expression «ce dist Lucans" qu'il suit pas à pas le texte latin. Il respecte la chronologie instaurée dans la narra-

instinctual forces that can only be construed as morally reprehensible », p. 173 \& ss. Le couple fonctionne ici cornme l'antithèse de celui formé par Pompée et Comélia, qui apparaît moralement exemplaire. Cette opposition réctuplique celle qui s'opère entre les deux héros, quant à la relation qu'ils entretiennent respectivement avec Rome: Pompée est le garant des libertés et des valeurs romaines, César est le conquérant, avide de pouvoir personnel.

${ }^{28} \mathrm{~A}$ la différence de sa source cependant, le compilateur porte un jugement certainement moins définitif, comme nous le verrons: les deux héros sont de fait plus ambigus.

${ }^{29}$ Dans ce passage, l'auteur réduit le sentiment amoureux à la seule attirance physique et ignore délibérément l'aspect sentimental et affectif, fidèle en cela à sa source. 
tion, et les figures féminines interviennent exactement au même moment du récit ${ }^{30}$, et sont investies des mêmes rôles. De même, il traduit, scrupuleusement le plus souvent, les dialogues et les discours qui s'apparentent à des monologues, mettant en scène de manière quasithéâtrale Marcia ou Cléopâtre et surtout Comélia, dont l'abondance des interventions directes accroît le pathétique. Il s'avère en outre respectueux des prises de position de Lucain. Celui-ci est très critique à l'égard de Cléopâtre et le compilateur d'abonder en son sens, soulignant parfois tel ou tel trait de caractère :

Car tant estoit plesanz et solaceuse et enlaçanz, que nus huem ne se poïst gaitier ne delivrer de li puis que il fust une nuit chaoiz en ses liens. (p. 638, 1. 9-11)

La répartition du blâme ou de l'éloge selon la figure féminine concernée va de pair avec les critiques très vives adressées à l'encontre de César et l'éloge continu de Caton et de Pompée. Le compilateur accentue les jugements portés par Lucain, se faisant simple commentateur ou observateur, par exemple à propos de l'adultère que commet César :

Molt l'em blasme Lucans. (p. 624, 1. 8)

Le passage du latin au français et du vers à la prose ne s'accompagne pas, semble-t-il, d'une perte de la matière originelle ou de son appauvrissement ni de son affadissement. Respectant la lettre et l'esprit du récit, le compilateur prend cependant bien des libertés qui confèrent à son œuvre le caractère d'une réécriture originale. D'évidence, son projet de dire l'histoire romaine est aussi guidé par une volonté de la rendre immédiatement accessible au public, que ce soit en vue de le divertir ou de l'instruire ${ }^{31}$. Et c'est pour combler l'écart entre avant et maintenant qu'il recourt aux figures de l'amplificatio ou de l'abbreviatio, et aux autres procédés éprouvés dans le roman ${ }^{32}$. Tout en leur conservant leur personnalité initiale, il présente les héroïnes de Lucain comme des héroïnes médiévales. Par rapport au texte latin, il introduit des détails, ou en modifie d'autres, qui réajustent l'image de la femme à la réalité du Moyen Age. Cette actualisation s'opère tantôt dans les scènes où elles sont impliquées, tantôt dans leurs interventions au style direct, tantôt dans leur portrait.

Le prosateur choisit parfois d'abréger les notations sur les rituels que développe la source. Il ne fait qu'évoquer le remariage de Caton et de Marcia, alors que Lucain en profite pour décrire le rituel romain ${ }^{33}$ :

Mes n'i ot onques semons parent ne ami, ne joie fete come l'en selt a noces. (p. 370, 1. 21-23)

Il préfère s'attarder sur l'aspect négligé de Caton et la tristesse des vêtements de Marcia. Il eût pourtant été intéressant, dans une œuvre à dessein didactique, d'avoir des informations sur une coutume qui n'est pas familière au public même letré. Ce laconisme est d'autant

\footnotetext{
${ }^{30}$ A quelques lignes près pour l'intervention de Marcia, voir note 6.

${ }^{31}$ B. Guenée insiste sur « la valeur éducative », sur « la valeur morale " et sur la « valeur d'exemple » qui sont propres à l'ouvrage et expliquent l'intérêt qu'il a suscité au cours du Moyen Age; c'est un «livre de sagesse ». Voir art. cit, p. 288.

${ }^{32}$ Pour une définition de ces termes, cf. Ed. Faral, op. cit. pp. 61-85.

${ }^{33}$ Lucain, II, 352-376.
} 
plus curieux que l'auteur insiste par ailleurs sur un autre rituel, celui de brûler les corps, à la mort de Pompée :

Tex estoit la maniere as enciens : l'en ardoit les cors et, selonc ce que chascuns estoit, l'en metoit ou feu armes et dras et espices, et les dames i metoient de lor chevex ; apres conquelloit on la cendre et l'estoioit l'en es temples ou em pyramides, tex conme est l'aguille dou borc seint Piere de Rome, ou en autres sarqeuz, selonc ce que chascuns avoit de pooir. (p. 584, I. 19-25)

Cette différence de traitement peut s'expliquer dans le premier cas par le souhait de focaliser le regard sur le caractère secondaire de ce remariage - par sa discipline rigoureuse, par sa sévérité envers la faiblesse humaine, le stoïcien Caton est détaché des contingences privées, et trop préoccupé par la situation de Rome pour accorder une grande importance à sa vie intime très liée à la vie publique ${ }^{34}$ - , dans le second cas par le rôle du rituel dans la narration - il permet en particulier à Comélia d'accomplir son devoir de parfaite épouse ${ }^{35}$.

On observe un autre type d'ellipse dans les scènes avec Cléopâtre, dont celle du festin. Le rituel et les habitudes égyptiennes sont négligés, l'auteur omettant certaines précisions comme celle des couronnes de nard et de rose posées sur la tête des convives ou encore celle de répandre sur les cheveux de l'amone et du cinname ${ }^{36}$. Dans la même scène, il pallie la suppression de tels détails par l'introduction de coutumes typiquement médiévales:

L'iaue fu demandee; l'en aporta et dona l'en as mains en bacins qui tot estoient de pur cristal. (p. 627, 1. 23-24)

Cléopâtre est une héroïne baignée dans un univers médiéval et occidental. La distance spatio-temporelle originelle d'avec le public se trouve occultée ${ }^{37}$. La démarche du compilateur est d'alléger sa narration, de la rendre plus alerte et plus vivante, et surtout immédiatement signifiante pour le public.

Le même souci l'anime dans la traduction des divers discours prononcés par les héroines. Les notations trop païennes sont remplacées par des termes chrétiens, dans la bouche de Cornélia :

« Ha ! li miens sire, ceste maudite, ceste esconmeniee vos a mort » (p. 565, 1. 14-15)

et même dans la traduction supposée directe de la source:

Maufé, ce dist Lucans, l'i aporterent, car il n'en vint onques se mal non et honte as Romains. (p. 623, 1. 17-18)

le traducteur attribuant à Lucain le terme de Maufé, en lieu et place de Erynnies. Des expressions sont ajoutées comme Ne place Dieu (p. 472, 1. 16-17). D'autres ressortissent à la féodalité comme seignorie de dame (p. 565, 1. 26), reine-lige (p. 624, 1.21). D'autres encore apparaissent, plus pittoresques et familières, et sans équivalent possible chez Lucain, dans la

\footnotetext{
${ }^{34}$ Voir J. M. A. Beer à ce sujet, op. cit. p. 176 et ss., qui montre en particulier comment le stoïcisme de Caton est récupéré par l'auteur médiéval et christianisé.

${ }^{35}$ Cf. éd. cit., p. 584, [14].

${ }^{36}$ Lucain, X, 155-171. De mème, il s'abstient de nommer les vins de Méróe et de Falerne (v. 163).

${ }^{37}$ Dans le même esprit,il fait un sort à l'onomastique romaine, négligeant de citer Fabricius, Curius et Cincinnatus (Lucain, X. 152-153).
} 
désignation de la coquetterie de Cléopâtre, avec des termes comme mercerie ou fere sa besoigne (p. 625, 1. 17-18).

Ces libertés montrent la distance prise avec la source, distance qui se retrouve en outre dans les conceptions énoncées chez Lucain et commentées et/ou réorientées par le traducteur. A propos du mariage de Caton et de Marcia, il est fait allusion à la morale chrétienne du mariage :

(Ici poent avoir grant honte meint crestien, cil meesmement qui ne prannent fames ne mes por lor luxure acomplir, quant Catons et auquant des Sarrazins n'orent fames ne mes por anfanz avoir). (p. 369, 1. 16-19)

Cette brève digression a un double impact : elle est l'occasion de faire à nouveau l'apologie de Caton tout en prenant l'allure d'une leçon de morale pour le public contemporain ou pour certains membres précis de ce public ? ${ }^{38}$ La portée critique de la traduction est également manifeste dans les choix du compilateur quant aux partis-pris de Lucain. Soit il les souligne, glosant par exemple la définition de la femme idéale:

* car se vos me mostrez amor en ma chetiveté et vos me suivez au fort et au dur, autresi come vos m'avez mostré senblant d'amor tant come je ai esté en ma prosperité et en $m^{\prime}$ onor, l'en en parlera a toz jors mes. (p. 547, 1. 23-27)

soit il les atténue pour en privilégier d'autres. C'est le cas avec le personnage de Cléopâtre. I reste réservé sur la mention de l'inceste, à l'encontre de Lucain qui accole systématiquement l'épithète d' «incestueuse » à la désignation de la reine ${ }^{40}$. Et s'il traduit les fortes imprécations de sa source contre Cléopâtre, il ne les prend quasiment jamais à son compte, prenant soin de renvoyer directement au poète latin. Il agit de même pour César, objet lui aussi de vives critiques chez Lucain, passant sous silence certaine appréciation dépréciative $^{41}$. A l'inverse, il est plus prolixe sur le pouvoir de séduction de la reine et sur son charme, inventant si besoin est:

Car tant estoit plesanz et solaceuse et enlaçanz, que nus huem ne se poïst gaitier ne delivrer de li puis que il fust une nuit chaoiz en ses liens. (p. 638, 1. 9-10)

Par ces retouches, le portrait de la reine est sensiblement modifié. L'accent est moins porté sur la perversion qu'est l'inceste que sur les capacités redoutables de la reine de perdre les hommes.

Le contenu de la source est transformé par un éclairage différent qui doit encore et surtout au recours des procédés d'écriture du roman ${ }^{42}$. Un riche usage des techniques

\footnotetext{
${ }^{38}$ Cela montre en même temps que pour le compilateur. la voluptas, rejetée et condamnée par les stoïciens, se réduit au seul plaisir sexuel. Cf. J. M. A. Beer, op. cit, p. 176.

${ }^{39}$ Le discours qu'il fait prononcer à Pompée est ainsi fait de répétitions absentes chez Lucain, VIII, 6385.

${ }^{40}$ Lucain, X, 107, 138.

${ }^{41}$ A la différence de Lucain (X, 169-170), il attribue par exemple, non au seul César, mais à l'ensemble des Romains, la volonté de conquérir l'Égypte par convoitise.

${ }^{42}$ Dans l'ouvrage s'observent aussi des empnunts aux techniques littéraires des chansons de geste, en particulier dans les scènes de bataille. B. Guenée en relativise l'usage : «notre historien n'est certes pas coupé de la culture et des goûts des laïques de son temps, mais avouons qu'il ne leur fait qu'un
} 
romanesques se manifeste dans les dialogues et monologues. En traduisant Lucain, l'auteur use de l'art du discours tel qu'il s'épanouit dans le roman. Le vers latin laisse place à une prose française travaillée et enrichie en fonction des nécessités inhérentes à une langue d'accueil. Ainsi, dans les discours, Comélia s'exprime-t-elle comme une héroüne de roman. Sa peine, sa révolte, son accablement sont traduits par la combinaison de la source et des figures de thétorique très en usage dans les romans en vers et en prose, comme le chiasme :

« La morz nos deüst departir, et tu nos deparz a la vie » (p. 473, 1. 9-10)

comme le jeu des oppositions et des antithèses qui assurent la cohésion et l'ossature du discours, jeu souvent relayé ou provoqué par les constructions symétriques et les effets de balancement :

«Cuides tu, se tu muers, que je voille vivre apres toi ? [...] autrement me convendroit vivre a honte et a dolor apres toi, car ja mes joie n'avroie. » (p. 473, 1. 22-25)

L'auteur utilise abondamment les synonymes qui confèrent une dynamique à la phrase, en même temps qu'ils précisent la pensée de l'héroïne et permettent de mettre en valeur la souffrance. Les interrogations oratoires parachèvent la charge pathétique du discours:

Por coi ? (p. 474, 1. 22)

Par ces différentes techniques, les prises de parole de Cornélia ressemblent étonnamment à celles d'héroïnes romanesques. Une similitude frappante, aussi bien par les procédés stylistiques que par la thématique, s'observe entre le monologue d'Hélène dans le Roman de Troie, à la mort de Pâris, et le discours où Comélia se juge coupable de la mort de Pompée ${ }^{43}$. Les paroles d'Hélène:

« Tant riche duc e tant bon rei

E tant riche amiraut preisié

En sont ocis e detrenchié !

Lasse ! a quel hore fui jo nee,

Ne por quei oi tel destinee

Que li monz fust par mei destruit ? (vv. 22930-22935)

« Ja nel deüssent consentir

Li deu ne la mer ne li vent. » (vv. 22984-22985)

trouvent un écho certain dans la lamentation de Comélia :

O Pompee, li miens sire, li mien amis, qui mout fusses dignes de meillor mariage que dou mien, se destinee chose fust, por quoi soffrirent onques li dieu que je fusse vostre, se fortune devoit avoir tel pooir en vos par mon pechié ? Ha! Lasse ! si mal fui

minimum de concessions. ", art. cit., p. 266. De fait, sur l'ensemble du texte, les techniques utilisées peuvent se compter. Et pourtant, elles jouent un rôle très important : elles révèlent la culture du compilateur, elles montrent comment il travaille et elles dévoilent les intentions qui l'animent, par sa manière de présenter et/ou d'orienter les faits.

${ }^{43}$ Cf. Benoit de Sainte Maure, Le Roman de Troie, éd. L. Constans, SATF, 6 vol., 19041912; traduction partielle par E. Baumgartner, 10/18, 1987. Sur ce monologue d'Hélène, voir notre article, "La complainte d'Hélène dans le Roman de Troie de Benoît de Sainte Maure (vv. 22920-23011)", Romania, 107, 1990, pp. 75-91. 
nee, quant tant poeple et tant roi sont trebuchié par moi ! Com mal vos reçui a mari, quant en tel maniere vos devoie fere chetif ! » (p. $548,1.30$, p. $549,1.2)$

La comparaison pourrait s'effectuer sur l'ensemble des deux discours, celui de Comélia, plus bref, étant assurément nourri du mode d'expression qu'est la complainte dans le roman en vers. Sans délaisser les données de la source, le compilateur se refuse donc à une traduction littérale, choisissant de présenter ses héroïnes comme des personnages de roman, peutêtre plus expressifs pour le public ${ }^{44}$.

Dans la narration, les techniques romanesques sont aussi largement requises, notamment dans la peinture des souffrances éprouvées par Cornélia. Le prosateur emprunte, semble-t-il, les images ovidiennes de la souffrance amoureuse qui sont monnaie courante dans les romans ${ }^{45}$. La douleur de Cornélia s'exprime, fût-ce pour des raisons différentes et avec davantage de sobriétét ${ }^{-6}$, comme celle de Didon dans l'Eneas ou comme celle de Laudine dans le Chevalier au lion de Chrétien de Troyes ${ }^{47}$ :

car ele [Cornelia] ne se pooit sostenir et s'aloit pasmant et roillant par mi la gravele dou rivage. (p. 475, 1. 2-3)

Ces notations demeurent cependant ponctuelles et fonctionnent surtout comme des réajustements destinés à rendre plus immédiate la réception de la prose française par le public.

Le compilateur va parfois plus loin lorsqu'il invente in extenso une description. Dans la représentation des figures féminines, le long portrait de Cléopâtre est l'exemple le plus achevé de la technique de l'amplificatio. Chez Lucain, nulle trace de prosopographie hormis quelques remarques sur la parure et les charmes de la reine ${ }^{48}$. L'examen de cette pause descriptive montre une application fidèle de l'art du portrait tel qu'il se pratique dans les romans et tel qu'il est enseigné dans les arts poétiques, sous la forme d'une succession de modèles ${ }^{49}$. L'auteur respecte l'ordre descendant de la description, avec la mention du visage en premier lieu, lui-même décrit de haut en bas :

\footnotetext{
${ }^{44}$ On pourrait objecter que le public qui serait initialement universitaire, déjà lettré et connaisseur du latin, pouvait se dispenser de semblables artifices. Mais l'écrivain - et donc son public-opéraient-ils, comme nous aujourd'hui, une distinction très nette entre les "genres", en l'occurrence roman et histoire, et le passage d'une langue à l'autre ne pouvait-il s'effectuer que de manière littérale? Et si la culture du compilateur est nourrie d'histoire, ne reposait-elle pas aussi sur la connaissance des ouvres autres, connaissance propre à tout clerc lettré?

${ }^{45}$ Dans les romans antiques comme dans les romans arthuriens, la peinture de la souffrance amoureuse est inspirée en grande partie d'Ovide. Sur ce point, voir Ed. Faral, Recherches sur les sources latines des contes et romans courtois du Moyen Age, Paris, Champion, 1913, p. 133 et ss. Voir aussi A. Petit, Naissances du roman. Les techniques littéraires dans les romans antiques du xIf siècle, Paris-Genève, Charnpion-Slatkine, 1985 , t. 1, p. 388 et ss.

${ }^{46}$ Il y a une sobriété évidente dans le texte en prose qui pourrait montrer que le compilateur a parfaitement assimilé et maitrisé les techniques habituelles et sait en doser l'usage. Pas de pathos excessif qui nuirait à l'effet recherché.

${ }^{47}$ Cf. Eneas, Roman du XIIf siècle, éd. J. -J. Salverda de Grave, Paris, Champion, 2 vol. , 1985-1989, vv. 1229 et ss. ; Chrétien de Troyes, Le chevalier au lion, èd. M. Roques, Paris, Champion, CFMA. $1982, v .1243$ et ss.

${ }^{48}$ Lucain, X, 136-143.

${ }^{49} \mathrm{Cf}$. les modèles de portraits masculins et féminins donnés par Matthieu de Vendôme dans son Ars versificatoria, Ed. Faral, Les arts poétiques..., p. 106 et ss.
} 
La cheveleüre ot sore et espesse et longue; le front large, plein et emple ; les sorcix gresles et voluz; les ielz pleins et vairs; le nes hautet et droit, de bele mesure; les orilles petites et netes; la bouche bien fete ; les levres un pou grossetes; le menton reont; la color freche et vermeille $*($ p. 626, 1. 30, p. 627, 1. 1-2)

S'ensuit la description du corps, beaucoup plus rapide comme de coutume quant il s'agit de femme $e^{50}$ :

Les mameles ot assez dures et petites, la jambe bien fete et le pié gent, petit et biau taillié. (p. 627, 1. 2-4)

L'auteur est en outre désireux de répondre aux canons de beauté en vigueur à son époque, ce qui ne manque pas d'être curieux pour dépeindre une Égyptienne. Les adjectifs utilisés en témoignent: Cléopâtre ressemble à Camille, à Polyxène, et à mille autres héroïnes romanesques par la blondeur de sa chevelure, par la clarté de ses yeux, par la blancheur de son teint avivé de rouge aux pommettes, par la minceur et les belles proportions de son corps... Le souci du détail est sensible dans la mention du menton, qui n'apparaît qu'au XII siècle chez Benoit de Sainte Maure, dans la prosopographie de Troilus ${ }^{51}$. Avant de dépeindre le physique de la reine, l'auteur décrit les vêtements, respectueux là encore des usages ordinaires dans les romans ${ }^{52}$. Il évoque sa parure, de la tunique au manteau, s'attardant sur les qualités de la matière et la richesse des accessoires:

Ele ot un fermaill a son col d'or et de genmes, qui tot li enluminoit la gorge, et une frenge lee de fil d'or environ la cheveçaille de sa porpre... (p. 626, 1. 19-21)

De sa source, il récupère habilement le détail des pierres précieuses trouvées dans la Mer Rouge $^{53}$. En ce portrait, l'auteur sacrifie au goût de ses contemporains pour de tels détails ${ }^{54}$ et il donne, à sa manière, une chronique de mode par les précisions sur la façon de porter les vêternents. Le traducteur s'efface momentanément devant le romancier, sinon devant un connaisseur averti des techniques romanesques.

Il n'en est pas pour autant asservi. Par certains écarts, il sait prendre ses distances par rapport à un modèle somme toute figé. Ainsi ne respecte-t-il pas intégralement l'ordre descendant de la description ${ }^{55}$. Il décrit le corps avant le visage pour terminer sur le corps. Suivant en cela l'ordre logique de sa description fondée sur l'attention portée aux vêtements, ce qui frappe au premier regard, il passe au corps par une transition cohérente :

\footnotetext{
${ }^{50}$ Voir les remarques d'Ed. Faral, Les arts poétiques, p. 80 et ss. ; cf. aussi notre ouvrage, Thèbes, Troie et Carthage, Poétique de la ville dans les romans antiques au XIf siècle, Champion, NBMA, 1994, p. 155 et ss. On observe un effort descriptif plus important pour le visage que pour le reste du corps, chose habituelle dans les portraits féminins. L'inverse se produit dans les portraits masculins où, en insistant sur le corps des hommes, les romanciers atténuent ce que la représentation du visage peut avoir de féminin.

${ }^{51}$ Benoît de Sainte Maure, op. cit, v. 5411.

${ }^{52}$ Il n'y a pas de règle particulière quant à la place des vêternents.

${ }^{53}$ Lucain, X, 130-140; Faits des Romains, p. 626, 1. 17-19.

${ }^{54} \mathrm{Le}$ public de clercs y trouve l'application des enseignements de techniques préconisées dans les arts poétiques et en usage dans les romans, et le public aristocratique un miroir -idéalisé ou rêvé -de luimême.

${ }^{55}$ Voir les remarques de J. M. A. Beer à ce propos, op. cit, p. 159.
} 
car ele ot les ataches [du manteau] ostees de son col por mielz mostrer la feture de ses espaules et de son cors, que ele ot assez bien mollee. (p. 626, 1. 26-27)

Il brise le carcan de l'ordre descendant, donnant un rythme vivant et naturel à une description toujours menacée de lourdeur et d'une convention sclérosante ${ }^{56}$. Surtout, il modifie les raisons d'être du portrait et son état d'esprit. Que l'amour soit lié à la beauté et que le portrait de la beauté le suscite immédiatement n'est pas une innovation. Le portrait explique et implique l'amour que va ressentir César pour Cléopâtre :

Parmi tot ice ele se fu fardee et apareillie, come cele qui voloit plere a Cesar, et come cele a cui la seignorie d'Egypte ne soffisoit pas, ainz tendoit a estre dame de Rome se ele le pooit enlacier. (p. 626,1.13-16)

L'originalité tient au déplacement de perspective. Alors qu'en règle générale, une équivalence s'instaure entre aspect physique et comportement moral, en l'occurrence entre beauté et bonté ${ }^{57}$, dans le cadre présent, elle s'opère entre beauté et mal ou perversité. La femme belle, la seule qui soit dépeinte comme telle dans le texte, est aussi une femme perverse et débauchée. L'insistance sur son maquillage et son fard, sur le soin accordé à sa toilette :

Tant s'estoit Cleopatra beau paree, que bel avival de puterie et de luxure avoit en li. (p. 627, 1. 9-10)

suffit à renverser la traditionnelle alliance entre beauté et bonté ${ }^{58}$. Alors que la beauté de Cléopâtre brûle César, elle s'avère froide et calculée. Au reste, les sentiments de la reine ne sont jamais vraiment exposés. Le personnage se sert de sa beauté et de sa sensualité comme César se sert de sa force pour parvenir à ses fins. Ainsi considéré, le portrait ne se réduit pas à l'une de ses fonctions habituelles - expliquer la naissance de l'amour-, il acquiert une vocation à la fois dramatique et «idéologique». Il justifie l'influence de Cléopâtre sur César et sur l'Histoire en général ${ }^{59}$, et il permet de dépeindre un type de femme fascinant, parce que dangereux mais attirant. Fidèle à Lucain, l'auteur ne manque pas de dénoncer cette perversité de la reine qui s'exerce sur les hommes, ce pouvoir exclusivement féminin, suspect car incontrôlable:

\footnotetext{
${ }^{56} \mathrm{Ce}$ risque a d'ailleurs été mentionné par les hétoriciens comme Geoffroy de Vinsauf qui, dans son Documentum de modo et arte dictandi et versificandi. préconisait, pour y échapper, de recourir au rajout de couleurs, de comparaisons ou d'images; cf. Ed. Faral, Les ants poétiques, p. 272 ; voir notre ouvrage, p. 182.

${ }^{57}$ Voir dans l'Ars versificatoria de Matthieu de Vendôme qui établit la relation entre éloge, beauté et bonté, et entre blârme, laideur et mal, Ed. Faral, Les arts poétiques, pp. 106-194. Cf. A. Colby, The portrait in Twelfth-Century French Literature. An exemple of the stylistic originality of Chretien de Troyes, Genève, Droz, 1965, p. 99.

${ }^{58}$ C'est chose assez rare pour le signaler d'autant que ce renversement donnera lieu à d'autres types d'alliances, comme celle qui se noue entre beauté et jeunesse en opposition à laideur et vieillesse, ainsi chez Adam de la Halle dans Le jeu de la Feuillée, CEuvres complètes, éd. et trad. de P. -Y Badel, Paris, Le livre de poche, Lettres Gothiques, 1995, p. 290 et ss.

${ }^{59} \mathrm{Cf}$. la célèbre pensée de Pascal : «Le nez de Cléopâtre s'il eût été plus court, toute la face de la terre aurait changé », Pensées, éd. de Ph. Sellier, Paris, Classiques Gamier, 1991, p. 163. Voir aussi p. 246.
} 
Bien dot l'en pardoner a Antoine ce que il fu puis sospris de s'amor, quant Cesar, qui tant avoit le cuer dur, en pot estre enlaciez... (p. 623, 1. 29-31)

Le portrait de Cléopâtre est complété et parachevé par la présentation du lieu du festin. A un individu débauché correspond un lieu et un peuple idoines. Le palais de Ptolémée et de Cléopâtre est propice à la luxure et à la mollesse :

Li leus meïsmes estoit covenables a la chose, car li pales estoit si beaus et si riches, ce estoit uns granz deliz a veoir. (p. 625, 1. 17-21)

Habitante et habitat sont tous deux source de perdition. La concordance semble aller de soi ; c'est peut-être l'une des raisons pour lesquelles le compilateur estime à deux ans - au lieu de six mois - le séjour de César en Égypte ${ }^{60}$.

Avec le portrait de Cléopâtre, l'auteur expose l'une de ses méthodes de travail. S'appuyant sur sa source - tous les éléments narratifs et descriptifs sont présents chez Lucain ou dans les gloses - , il utilise parallèlement les techniques romanesques au service de l'Histoire, mais en leur ôtant leur caractère figé ou conventionnel. Il redéfinit notamment la configuration du beau, en bouleversant les schémas pré-établis, en appliquant à la beauté la dimension de blâme déterminée par Edmond Faral. Marquant ses distances à la fois avec le texte-source et avec les romans où il puise, il introduit l'ambiguité en gommant le caractère lisse et uniforme des héroïnes belles et bonnes, et donne à Cléopâtre une réelle profondeur psychologique. Cette femme, belle et coquette, est un personnage complexe et ambigu, digne pendant de César, lui aussi héros ambigu et à multiples facettes ${ }^{61}$. L'historien ne condamne jamais les héros, de son propre chef. Quand sa source l'y contraint parce qu'il se veut fidèle, il prend soin de se réfugier derrière elle, sans témoigner de son adhésion à ce qui est dit.

Le prosateur maîtrise à l'évidence les procédés romanesques, et leur usage dans un texte à vocation historique s'accompagne d'un grand intérêt pour une thématique privilégiée dans les romans, celle de l'amour, marginale chez Lucain, fût-ce dans la représentation des figures féminines. Par rapport aux vers latins, l'auteur glose, transforme ou ajoute, tout particulièrement avec les personnages de Comélia et de Cléopâtre. Ces deux figures symbolisant la complexité féminine entrent en effet dans des problématiques amoureuses différentes. Comélia est déchirée entre son amour et les exigences qui en découlent, et son sens du devoir et de l'obéissance à l'époux. Ses discours reflètent une dialectique impossible à résoudre, d'autant qu'en face d'elle, Pompée est la voix de la raison ou plus exactement choisit la raison lorsqu'il est confronté au même dilemme. Cléopâtre ne connait pas les tourments de l'amour, mais elle les inflige à César dont elle est finalement aimée ${ }^{62}$ :

${ }^{60} \mathrm{Cf}$. p. 623, l. 24.
${ }^{61}$ Voir le portrait que le compilateur fait à la fin du récit : il décrit aussi bien le physique que le caractère
et les moeurs de César; éd. cit., p. 718 et ss. G. Spiegel pense que le portrait de César est totalement
négatif (" a piteous figure deranged by his pride and desire for honor ", " the wrathful bellator of heroic
epic, whose violent rage knows no bounds...") et s'oppose à Pompée, modèle de probité, d'honnêteté,
de modestie et de mesure, le véritable garant des valeurs romaines, op. cit., p. 172. On trouve
assurément tous ces traits chez César, mais on trouve aussi l'intelligence, le sens de la stratégie, la
pugnacité, le courage... et une capacité à aimer... Sur César, perçu au contraire comme un héros
médiéval modèle, voir l'étude de M. A. J. Beer, op. cit
${ }_{62}$ Même si le compilateur émet des objections sur cet amour, en expliquant que César est resté deux ans
en Égypte, non pour Cléopâtre mais pour entendre l'enseignement d'Achoreus (p. $629,1.7-12$ ). Les 
car il l'amoit; et ne fust por amor de li, il eüst fet province dou regne d'Egypte. (p. $656,1.20-21)$

L'amour est une arme dont elle se sert pour convaincre et vaincre, et pour gouvemer. Dans cette double configuration où l'une aime et est victime de l'amour par le destin, et où l'autre est aimée et maîtresse de ses sentiments, les deux femmes ne sont pas forcément opposées ou antithétiques.

Elles incament le double visage de la femme, objet de fascination. La femme est à la fois le Bien et le Mal. Comélia et Cléopâtre en sont les vivants témoins par des moyens différents. En tant qu'épouse, Comélia est soutien de son mari, source de vie par la matemitée ${ }^{63}$ et relais dans la transmission du testament moral et politique du père au fils. L'adresse à Sextus est explicite :

"Tu, Sexte, beaus amis, pren les enseignes ton pere, porte les par le monde, met toi es avantures des batailles. Pompee me conmanda que je te le deïsse. " (p. 581, 1. 14-16)

En même temps, elle est celie par qui le malheur arrive. Elle répète inlassablement dans ses discours qu'elle est la cause de la perte de son époux, nouvelle Hélène qui s'apitoie sur le sort de son second mari ${ }^{64}$ :

«Ge ai II. foiz neü au monde, car je sai bien que mes pechiez et ma meschaance ont fait par quoi li mondes a perdu tex governeors com fu Crassus, qui fu ocis des Turs, et con estoit Pompees, qui veincuz est de Cesar. » (p. 548, 1. 23-27)

Le péché dont elle s'accuse - son second mariage ou plus largement sa nature de femme? - la qualifie de femme maléfique qui suscite perdition et mort. L'échec de Pompée n'est plus alors causé par la supériorité militaire et tactique du chef ennemi, César, mais par une femme et la faute morale que constitue un remariage hâtif ${ }^{65}$. L'histoire privée de Pompée déteint en définitive sur son histoire publique au point de circonvenir celle-ci. Sans doute peut-on voir dans cette auto-accusation de Comélia, volontiers développée par Lucain, une intention précise : laisser à Pompée mort toute sa grandeur et sa gloire passées en déplaçant sur un mariage malheureux les conditions de l'échec. N'en demeure pas moins une image de la femme qui repose presque toujours sur la représentation d'un personnage bi-frons. De manière plus évidente, Cléopâtre est une véritable dévoreuse d'hommes et Lucain d'énumérer ses amants et maris, Ptolémée, César et Antoine (p. 623, 1. 24-26). Le prosateur reprend au texte latin la comparaison avec Hélène, tout en précisant que Cléopâtre n'entraine pas la chute de Rome, à la différence de l'hérö̈ne grecque qui cause la chute de Troie. La comparaison semble inadaptée eu égard aux personnalités si différentes des deux figures féminines, le seul point commun étant la beauté qui est sujette à la perte de l'homme. Hélène subit son

éditeurs voient dans ces propos l'écho d'une discussion d'école, éd. cit., t. 2, p. 204. Cela montre en tout cas que, pour le compilateur, la problématique amoureuse est omniprésente dans cet épisode, quelles qu'en soient les formes ou les variations.

${ }^{63}$ Mais c'est Marcia qui est l'exemple le plus significatif de cette vocation maternelle.

${ }^{64}$ L'Hélène du Roman de Troie, bien sûr, et non celle que le compilateur évoque uniquernent pour sa beauté destructrice dans une comparaison avec Cléopâtre: voir éd. cit., p. 623, 1. 18. Sur l'autoculpabilisation de Comélia, cf. aussi p. 565 [21].

${ }^{65}$ Le songe de Pompée est révélateur. Julia lui adresse directement des reproches, à lui mais aussi à Comélia : p. 387, 1. 2 et ss. 
destin, Cléopâtre le provoque. La démonstration reste toutefois la même: la femme et l'amour décident généralement du destin politique et militaire des hommes, qu'ils le favorisent ou y fassent obstacle.

La réflexion sur l'amour est parachevée d'une réflexion sur le mariage, à travers le couple Pompée/Comélia. Ce demier incarne l'une des apories majeures soulevées dans la littérature romanesque du Moyen Age, dans le roman arthurien en particulier ${ }^{66}$, le conflit entre amour et prouesse, transposé ici dans le cadre du mariage, d'où une interrogation subséquente sur l'amour dans le mariage:

« et ge dor chascune nuit delez ma fame autresi a seür con se la bataille ne devoit pas estre. Quelx honors seroit ce quant cor et boisines soneront por tot le monde assembler a bataille, se ge me vouloie lever del giron ou del lit ma fenme por aler en assaut ? »( p. 472, 1. 20-24)

Dans les Faits des Romains, le mariage d'amour est un obstacle, non pas à la prouesse guerrière en tant que telle, mais à la conduite digne d'un chef militaire et politique : le devoir de Pompée comme époux et l'amour qu'il voue à sa femme ont en effet interféré, aussi peu que ce soit, dans ses décisions politiques et militaires. La question de l'amour dans le mariage n'est point résolue puisque Pompée meurt de manière peu glorieuse, assassiné par des sbires de Ptolémée, sous les yeux de son épouse impuissante et repentante. Et la seule réponse, peu exaltante, que livre le texte est l'exemple de Caton et de Marcia, le seul couple dans lequel les sentiments ont peu de place et ne peuvent constituer une entrave au destin public de Caton.

$\mathrm{Par}$ la seule convocation de techniques romanesques, le compilateur tire parti de sa source en brossant des figures féminines qui deviennent de véritables personnages de roman. Autour d'elles s'élabore une réflexion sur l'amour et le mariage, en relation avec le pouvoir, avec ses conditions de succès et d'échec. D'un épisode historique où leur rôle est apparemment négligeable - ce qui reste le cas de Marcia - et où elles servent surtout à figurer la vertu et le vice, le bien et le mal, elles deviennent dans le texte vernaculaire des consciences individualisées qui sont autant d'interrogations et de remises en cause de l'univers masculin.

Fidèle à son dessein de dire et de traduire l'histoire romaine, l'auteur des Faits des Romains est respectueux de sa source principale, ici Lucain, dans la représentation des figures féminines. C'est l'occasion de dépeindre trois femmes, Marcia, modèle d'épouse et de mère parfaites, Comélia, épouse aimante et soumise, enfin Cléopâtre, le contre-modèle, la femme fatale, perverse et avide de pouvoir. Pour favoriser la réception de ces trois figures par le public médiéval, le prosateur choisit de recourir à des procédés romanesques, dont celui du discours et celui de l'amplificatio par le biais du portrait. Leur usage aboutit à une réorientation des données du texte latin; il permet de brosser des femmes saisies dans le doute, dans la souffrance, ou dans l'action, de les faire vivre comme des personnages de roman. De fait, l'image qui se dégage d'elle est complexe et ambiguë; elle reflète la perception, à la fois maléfique et bénéfique, de la femme inhérente à l'imaginaire

\footnotetext{
${ }^{66}$ Et notamment chez Chrétien de Troyes, dans Le Chevalier au lion, par exemple avec le couple Yvain/Laudine.
} 
médiéval $^{67}$, et permet de poser nombre d'interrogations sur l'amour, sa place dans le mariage et dans la société, et son rôle dans la conquête et dans la conservation du pouvoir. Le respect de la source et l'insertion d'une actualité romanesque par des techniques et une thématique déterminent les termes de la vocation historique de l'ouvrage: histoire sans doute, histoire romancée sans aucun doute.

Catherine Croizy-Naquet (Université de Paris III)

${ }^{67}$ Pas seulement médiéval, d'ailleurs. En témoigne le traitement que Lucain lui réserve tout au long de son poème et dont s'inspire le compilateur. 